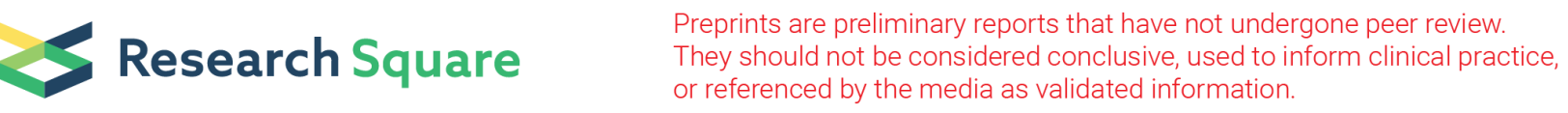

\title{
WNK1 Antagonist Decreased Sevoflurane-induced Neurotoxicity in HT22 Hippocampal Neurons via the WNK1/NKCC1 Signalling Pathway
}

\section{Ya-Fan Bai}

Capital Medical University Affiliated Beijing Friendship Hospital Department of Anesthesiology https://orcid.org/0000-0003-2449-4909

\section{Wen-Jing Li}

Capital Medical University Affiliated Beijing Friendship Hospital Department of Anesthesiology

\section{Li Zhang}

Department of anesthesiology, Beijing Friendship Hospital, Capital Medical University

\section{Jun-Fa Li}

Capital Medical University Department of Neurobiology and Bejing Institute for Brain Disorders

Li-Xin An ( $\square$ anlixin8120@163.com )

https://orcid.org/0000-0001-6344-4010

\section{Research article}

Keywords: WNK-463, sevoflurane, neurotoxicity, WNK1/NKCC1 pathway

Posted Date: October 21st, 2020

DOl: https://doi.org/10.21203/rs.3.rs-29017/v3

License: (c) (1) This work is licensed under a Creative Commons Attribution 4.0 International License. Read Full License 


\section{Abstract}

Background: The $\mathrm{GABA}_{A} \mathrm{R}$ plays an important role in the neurotoxicity caused by sevoflurane, but the mechanism of its regulation in this context is unknown. The present study aimed to reveal the WNK1/NKCC1 pathway as a regulator of the neurotoxicity caused by sevoflurane on the HT22 hippocampal neuron cell line.

Methods: HT22 cultured hippocampal neurons were divided into three groups: control group, 4.1\% sevoflurane treatment group for $6 \mathrm{~h}$, WNK-463 (specific antagonist of WNK1, $1 \mu \mathrm{mol}$ ) + sevoflurane treatment group. Cell viability and the optimum concentration of WNK-463 were measured by MTS method. Cell apoptosis was detected by Tunel method, and the content of cleaved caspase-3 protein apoptosis factor was detected by Western blot. Pathway protein detection including the expression of WNK1, NKCC1 were detected. Calcium imaging measures intracellular calcium ion concentration and verifies downstream targets.

Results: The neurotoxic effects of sevoflurane on hippocampal neurons were observed. As setting the results of control group is " 1 ", compared with sevoflurane group, WNK-463 downregulated the protein expression of cleaved caspase-3 ( $1.96 \pm 0.49$ vs $1.29 \pm 0.38)$, increased cell viability ( 0.68 vs 0.96$)$ and decreased apoptosis $(13.31 \pm 4.67 /$ vision vs $7.05 \pm 3.82 /$ vision, $\star \star \star P<0.001)$. Compared with the control group, sevoflurane treatment increased the expression of WNK1 kinase and NKCC1 protein, whereas WNK-463 reversed this increase without affecting the control HT22 cells $\left({ }^{\star} P<0.05\right)$. Compared with the control group, sevoflurane exposure in HT22 cells increased intracellular $\mathrm{Ca}^{2+}$ concentrations, while WNK463 reversed this change ( 1.0 vs $2.08 \pm 1.36$ vs $1.26 \pm 0.77$, $* * P 0.01$ ).

Conclusion: This study demonstrated a neuroprotective role of the WNK1 antagonist WNK-463 in sevoflurane-induced neurotoxicity. WNK-463 promoted hippocampal neuron viability and reduced the apoptosis and intracellular calcium overload caused by sevoflurane on HT22 hippocampal neurons, possibly via the modulation of the WNK1/NKCC1 pathway.

\section{Background}

The neurotoxic effect caused by general anaesthesia in the developing brain has caused continual concern. Numerous animal studies have found that an exposure to most general anaesthetics in mammals at their peak of development leads to a range of morphological changes ${ }^{[1-3]}$. These changes include neurodegenerative changes ${ }^{[3,4]}$, neural apoptosis or cell death, and impaired neurogenesis ${ }^{[5]}$. In addition, anaesthesia exposure in infancy has also been associated with memory impairment, behavioural disorders, and poor intellectual development persisting to early adulthood ${ }^{[6-10]}$. Repeated and prolonged exposure to general anaesthetics in the developing brain is still a high-risk factor for neuronal injury ${ }^{[6,11,12]}$. Therefore, it is of great significance to explore the mechanism of neurotoxicity caused by general anaesthetics in developing neurons. 
The $\gamma$-aminobutyric acid type $A$ receptor $\left(G A B A_{A} R\right)$ plays an important role in the mechanism of general anaesthesia ${ }^{[13,14]}$. Sevoflurane, a representative inhaled general anaesthetic, stimulates $G A B A_{A} R$ to induce the flow of chloride ions $\left(\mathrm{Cl}^{-}\right)^{[14]}$. In the adult brain, $\mathrm{GABA}_{A} \mathrm{R}$ activation triggers $\mathrm{Cl}^{-}$flowing into cells, membrane hyperpolarization and synaptic inhibition. Conversely, in the developing brain, $G A B A_{A} R$ activation triggers $\mathrm{Cl}^{-}$flowing out of cells, membrane depolarization and synaptic excitation ${ }^{[15,16]}$. This developmental "switch" in $\mathrm{GABA}_{A} \mathrm{R}$ function from excitability to inhibition has been attributed to the difference in the $\left[\mathrm{Cl}^{-}\right] \mathrm{i}$ of immature versus mature neurons ${ }^{[16,17]}$. This difference in $\left[\mathrm{Cl}^{-}\right]$i at different stages results from the conversion of receptors with a dominant function from $\mathrm{Na}^{+}-\mathrm{K}^{+}-\mathrm{Cl}^{-}$cotransporter 1 (NKCC1) to $\mathrm{K}^{+}-\mathrm{Cl}^{-}$cotransporter $2(\mathrm{KCC} 2)^{[17]}$.

WNK (with no lysine) kinases are $\mathrm{Cl}^{-}$-sensing kinases, including WNK1, that regulate the activities of NKCC1 and KCC2 [17]. In our previous study, we found that sevoflurane's neurotoxicity on the developing brain was related to the changes in the transition of $\mathrm{GABA}_{A} \mathrm{R}$ a1 and $\mathrm{a}_{2}{ }^{[18]}$. This neuronal damage was decreased by the NKCC1 inhibitor bumetanide ${ }^{[18]}$. Stevens ${ }^{[19]}$ also found that an NKCC1 inhibitor prevented ketamine neurotoxicity. Therefore, we hypothesized that when a WNK1 inhibitor (WNK-463) is administered, NKCC1 activity can be downregulated, the concentration of chloride ions in cells can be reduced, and the excitatory neurotoxic effects caused by sevoflurane can be decreased in the developing brain.

As the research object, hippocampal neuronal HT22 cell line from mouse ${ }^{[20]}$ were used to verify the above hypothesis and explore the neurotoxicity mechanisms of sevoflurane on neurons, with the aim to provide new ideas for ameliorating the neurotoxicity of inhalational anaesthetics.

\section{Materials And Methods}

\subsection{Cell culture}

The hippocampal neuronal HT22 cell line of mouse were purchased from the American Type Culture Collection (ATCC). HT22 cells were maintained in Dulbecco's modified Eagle's medium (Gibco, Grand Island, USA) containing 10\% foetal bovine serum (Gibco, Grand Island, USA) and 1\% penicillinstreptomycin solution (Life Technologies, Carlsbad, ON, Canada) at $37^{\circ} \mathrm{C}$ in a humidified incubator with $5 \% \mathrm{CO}_{2}$. The medium was completely changed every $72 \mathrm{~h}$.

All HT22 cell lines were divided into three groups. The control group, sevoflurane treatment group and sevoflurane-WNK-463 group. The cell culture was exposed in an airtight plastic chamber with inlet and outlet connectors. The inlet port of the chamber was used to adjust the concentration of sevoflurane (AbbVie Inc., North Chicago, IL, USA), which was connected to a sevoflurane vaporizer. The outlet of the chamber was used to monitor sevoflurane concentration through a gas monitor (PM 8060, Drager, Lübeck, Germany) until the target concentration was reached. 
In the control group, the cell culture was grown in the chamber with humidified atmosphere with $5 \% \mathrm{CO}_{2}$ $\left(95 \%\right.$ air $\left./ 5 \% \mathrm{CO}_{2}\right)$ at $37^{\circ} \mathrm{C}$ for $6 \mathrm{~h}$.

In the sevoflurane group, the chamber was gassed with a concentration of sevoflurane $(4.1 \%)$ in the carrier gas $\left(95 \%\right.$ air $\left./ 5 \% \mathrm{CO}_{2}\right)$ for $30 \mathrm{~min}$ as described previously ${ }^{[21]}$. And the cell culture was put in the chamber and then kept tightly sealed for $6 \mathrm{~h}$ at $37^{\circ} \mathrm{C}$.

The WNK1 inhibitor WNK-463 was dissolved in dimethyl sulfoxide (DMSO) and diluted with sevofluranetreated medium. In the sevoflurane-WNK-463 group, the cell culture was pre-treated with WNK-463 in the medium for 30 mins, and then treated with sevoflurane $(4.1 \%)$ in the carrier gas $\left(95 \%\right.$ air $\left./ 5 \% \mathrm{CO}_{2}\right)$ for $6 \mathrm{~h}$.

\subsection{MTS assay}

The CellTiter 96® AQueous One Solution Cell Proliferation Assay Kit (Promega Corporation, Madison, WI, USA) is a colourimetric method for determining the number of viable cells and screening of optimum drug concentration (WNK-463) in proliferation in cytotoxicity assays according to a previous study ${ }^{\text {[22] }}$. We added WNK-463 at a certain concentration gradient of $0,0.5,1.0,2.5,5.0 \mu \mathrm{mol}$, in order to find the optimum concentration of WNK-463 through the results of MTS. Move $20 \mu \mathrm{l}$ of CellTiter $96 \AA$ AQueous One Solution Reagent into each well of the 96 -well assay plate containing the samples in $100 \mu$ l of culture medium. The culture wells with MTS reagent was placed in an incubator at $37^{\circ} \mathrm{Cfor} 2$ hours and then recording the absorbance at $490 \mathrm{~nm}$ with a 96-well plate reader. The results of the control group were set at $100 \%$ and the survival rates of the other two groups were calculated. From the MTS results (absorption was detected at $490 \mathrm{~nm}$ using a microplate reader), we found that with the increase in WNK-463 concentration, the protective effect was gradually enhanced and reached a peak at $1.0 \mu \mathrm{mol}$ before it decreased which was used in our subsequent experiments $(P<0.01$, see supplementary file, S-Figure 1$)$.

\subsection{TUNEL assay}

The terminal deoxynucleotidyl transferase (TdT) dUTP nick-end labelling (TUNEL) method was performed to label the 3'-terminus of the fragmented DNA of apoptotic cells according to the manufacturer's protocol ${ }^{[20]}$ (One Step TUNEL Apoptosis Assay Kit, Beyotime, China). Phosphate-buffered saline (PBS) containing 4\% paraformaldehyde was added to the three groups of cells and incubated for 1 hour and washed with $1 \times$ PBS 3 times for 5 min. After permeabilization with $1 \times$ PBS containing $0.1 \%$ Triton X-100 for $2 \mathrm{~min}$ on ice, the cell slices were incubated with a TUNEL working solution for $1 \mathrm{~h}$ at $37^{\circ} \mathrm{C}$ in darkness. The TUNEL-positive cells were labelled by Cy3 and were examined with fluorescence microscopy (Olympus IX710 Camera, Japan). The cells with green fluorescence were considered apoptotic cells. To count the number of apoptotic cells, we selected the same-sized areas of each cell slice for analysis with Image Pro Plus software.

\subsection{Measurement of intracellular calcium levels ([Ca $\left.{ }^{2+}\right]$ i)}


According to before the treatment method of cells after collecting, learned that, the supernatant fluid to join without phenol red culture medium, Hank's balanced salt solution (HBBS) wash three times, to join the Fluo-4, AM concentration of 5 umol/L working liquid Beyotime biological co, LTD(Shanghai), incubation in $37^{\circ} \mathrm{C}$ for 30 min, using HBSS flushing cells 3 times, and continue in HBSS incubation for $30 \mathrm{~min}$, under laser confocal microscope test, excitation wavelength of $494 \mathrm{~nm}$, emission wavelength of $516 \mathrm{~nm}$.

\subsection{Western blot analysis}

The proteins including caspase-3, WNK1, NKCC1 were detected by western blot analysis. The proteins were extracted using RIPA lysis buffer (Beyotime Biotechnology, Shanghai, China). The protein concentrations were quantified using the $\mathrm{BCA}^{\mathrm{Tm}}$ Protein Assay Kit (Pierce, Appleton, WI, USA). Equal amounts of protein were resolved over $8-12 \%$ sodium dodecyl sulfate polyacrylamide gel electrophoresis (SDS-PAGE) and transferred onto polyvinylidene fluoride (PVDF) membranes. The membranes were incubated in $5 \%$ non-fat milk for $1 \mathrm{~h}$ at room temperature and then incubated with primary antibodies overnight at $4^{\circ} \mathrm{C}$. The primary antibodies were caspase-3 antibody (\#9662, 1:1000 dilution), WNK1 antibody (NB600-225, 1:500 dilution) and NKCC1 antibody (\#14581,1:500 dilution). The membranes were then incubated with the secondary antibodies (Abcam) for $1 \mathrm{~h}$ at room temperature. Positive signals were visualized by enhanced chemiluminescence (ECL) reagent (GE Healthcare, Little Chalfont, UK). The intensity of the bands was quantified using Image Lab ${ }^{\text {TM }}$ Software (Bio-Rad, CA, USA).

\subsection{Statistical analysis}

All experiments were repeated three times. The results of multiple experiments are presented as the means \pm standard deviations (SD). Statistical analyses were performed using GraphPad Prism 7 (GraphPad Software Inc., San Diego, CA, USA). We used one-way variance analysis (ANOVA) to compare the overall difference, and Posthoc test analysis to compare the differences between two groups. The $P$ values were calculated using Posthoc test analysis. A $P$-value of $<0.05$ was considered to indicate statistical significance.

\section{Results}

\subsection{WNK-463 alleviated the sevoflurane-induced reduction in cell activity}

The MTS method was used to detect hippocampal neuronal HT22 cell activity. The I control group was $100 \%$ and that of the sevoflurane group was $75.40 \%(P<0.0001$, S-Figure 2$)$. According to the results of different concentration of WNK-463 on MTS (S-Figure 1), $1.0 \mu$ mol WNK-463 was the optimum concentration which was used in the next experiment. Compared to control group, sevoflurane obviously reduced $\mathrm{HT} 22$ cell activity, and $1 \mu \mathrm{mol}$ WNK-463 showed a significant protective effect $(P=0.006$, Figure 1).

\subsection{WNK-463 decreased the HT22 cell apoptosis caused by sevoflurane}


The results of TUNEL, in the control group, the positive cells were $4.44 \pm 2.53 / \mathrm{mm}^{2}$ through the observation of the field of fluorescence microscope, whereas in the sevoflurane group the positive cells were $13.31 \pm 4.67 /$ vision. WNK-463 decreased the positive apoptosis cells compared with sevoflurane group (7.05 $\pm 3.82 /$ vision vs $13.31 \pm 4.67 /$ vision) $(P<0.001$, Figure $2 \mathrm{~A}, \mathrm{~B}, \mathrm{C}, \mathrm{D})$.

Through the detection of caspase-3, the results indicated that cleaved caspase-3 protein was significantly upregulated in cells after sevoflurane treatment compared to the control cells. Moreover, cleaved caspase- 3 activity was significantly reduced after WNK-463 treatment compared to the sevoflurane group $(P=0.034$, Figure $2 \mathrm{~F})$.

\subsection{WNK-463 inhibited the WNK1 kinase concentration increase caused by sevoflurane}

To explore the mechanism of apoptosis and the protective mechanism of the WNK1 inhibitor, the expression levels of WNK1 kinase proteins were investigated in HT22 cells after treatment with sevoflurane. The results indicated, that the WNK1 kinase proteins in cells in the sevoflurane group were significantly upregulated ( $P<0.001$; Figure 3 ). There was no significant difference between the WNK-463 group and the control group. However, after exposure to sevoflurane, the WNK1 concentration was significantly increased, and this high expression could be inhibited by WNK-463.

\subsection{WNK-463 inhibited the NKCC1 concentration increase caused by sevoflurane}

To verify the role of WNK1/NKCC1 signalling pathway, the expression levels of NKCC1 proteins were investigated in HT22 cells after treatment with sevoflurane. The results indicated, compared with those in the control group, that the NKCC1 proteins in cells in the sevoflurane group were significantly upregulated after exposure to sevoflurane, and this high expression could be inhibited by WNK-463. ( $P=0.031$; Figure 4).

\subsection{WNK-463 reduced the calcium overload in HT22 cells after exposure to sevoflurane}

Calcium ion imaging technology (Fluo-4) was used to detect the basic values of calcium in the cells. The Fluo-4 images showed that the fluorescence intensity of the sevoflurane treatment group was significantly enhanced compared to that of the control group. However, compared with the sevoflurane group, the WNK-463 treatment group showed a weak fluorescence intensity differ from the control group (Figure 5A, B, C), indicating that the WNK1 inhibitor reduced the calcium overload caused by sevoflurane. The results indicated, compared with those in the control group, that the calcium ion in cells in the sevoflurane group were significantly upregulated $(P<0.001$; Figure 5D).

\section{Discussion}

Our study showed that the hippocampal neuronal HT22 cell had lower cell activity and higher apoptosis rate after exposure to sevoflurane. These changes related to the overload of intracellular calcium and high expression of WNK1 kinase. However, the WNK1 inhibitor WNK-463 could improve cell activity, 
decrease the apoptosis caused by sevoflurane, downregulate the expression of WNK1/NKCC1 and alleviate calcium overload. Therefore, we propose that the WNK1/NKCC1 signalling pathway may play a major role through intracellular calcium overload in sevoflurane-induced neurotoxicity, which may be a potential protective mechanism against sevoflurane-related neurotoxicity.

Most general anaesthetics are excitants of $G_{A B A} R$ and inhibitors of the N-methyl-D-aspartate (NMDA) receptor. Currently, many studies demonstrated that neurotrophic apoptosis ${ }^{[2,3]}$, mitochondrial damage and accumulation of ROS ${ }^{[23]}$, inflammatory factors ${ }^{[16,24]}$, the excitatory neurotoxicity ${ }^{[3]}$, the effects on the regeneration of neurons, changes in receptor subtype ${ }^{[18]}$ were the possible mechanisms of general anaesthetics' neurotoxicity ${ }^{[19-25]}$. In the present study, we found that exposure of sevoflurane caused neuron apoptosis and decreased cell vitality, which was consistent with the above conclusions.

In the present study, we found that WNK-463, an inhibitor of WNK1 kinase, may play a protective role in developing neurons. WNK1 kinase is a $\mathrm{Cl}^{-}$-sensitive serine threonine protein kinase expressed in the central nervous system. Affected by intracellular chloride ions, this kinase can directly inhibit KCC2 activity and enhance NKCC1 activity ${ }^{[17]}$. According to the level of intracellular chloride ion concentration, WNK1 kinase negatively regulates intracellular $\mathrm{Cl}^{-}$concentration by inhibiting its downstream $\mathrm{KCC} 2$ and raise NKCC1 channel protein, maintaining intracellular osmotic pressure. WNK1 kinase directly on $\mathrm{KCC} 2 / \mathrm{NKCC} 1$. It also activates the SPS1-related proline/alanine rich kinase (SPAK) and the related oxidative stress response protein OSR-1 (OSR-1), namely, those fighting-regulating the endogenous neural cells [ $\left.\mathrm{Cl}^{-}\right]$through the WNK1-SPAK-OSR-KCC2 /NKCC1 pathway, then the oxidative stress affected by the oxidative stress of SPS1-related proline/alanine rich kinase (SPAK) and those related to it. Furthermore, it affects the depolarization of $G_{A B A} R$ during excitation of developing neurons, and transforms to inhibitory function ${ }^{[25-27]}$. Friedel ${ }^{[28]}$ found that in the process of neuron development, GABA $A$ is open, which transforms from the excitatory effect of immature neurons to the inhibitory effect of mature neurons, and an important guaranteed factor is that as $\mathrm{KCC} 2$ transports $\mathrm{Cl}^{-}$synergistically out of cells, [Cl-]i decreases. As an inhibitor of NKCC1, bumetanide can attenuate the increase in apoptosis induced by sevofluran $e^{[18,29]}$. Stevens ${ }^{[19]}$ also found that the NKCC1 inhibitor alleviated the decline in the learning and memory function of newborn rats caused by ketamine. Therefore, we propose that as a cotransporter of NKCC1 and KCC2, WNK1 can affect the transformation of NKCC1-mediated $\mathrm{Cl}^{-}$flow and affect the excitability of $\mathrm{GABA}_{A} \mathrm{R}$ in the immature brain. In the present study, we found that sevoflurane caused a significant decrease in cell activity and an increase in apoptosis and cleaved caspase-3 expression in the hippocampal neuronal HT22 cells of neonatal rats. After the administration of the WNK1 inhibitor WNK463 , cell activity was improved, and apoptosis and the expression of cleaved caspase-3 were decreased. These results demonstrated that the WNK1 inhibitor was a protective factor against the neuroexcitatory toxicity of sevoflurane in developing neurons, which is consistent with our hypothesis.

We also found that sevoflurane exposure caused calcium overload in HT22 cells. In addition, the calcium current generated by the NMDA receptor directly activates voltage-dependent $\mathrm{Ca}^{2+}$ channels (VDCCs), 
leading to increased intracellular $\mathrm{Ca}^{2+}$ and calcium overload. Calcium is an important regulator, and intracellular $\mathrm{Ca}^{2+}$ overload can lead to apoptosis through the activation of apoptosis-related enzymes, such as phospholipase, protease, and nucleic acid enzymes, as well as mitochondrial and cell membrane damage, and the generation of a large number of free radicals ${ }^{[30]}$. In the present study, the results of the Fluo-4 imaging of intracellular calcium showed a significant accumulation of $\mathrm{Ca}^{2+}$ in $\mathrm{HT} 22$ cells after treatment with sevoflurane. WNK1 inhibitor administration significantly decreased the calcium density, which demonstrated that the WNK1 inhibitor may influence NKCC1 and then $\mathrm{GABA}_{A} R$ excitability and intracellular $\mathrm{Ca}^{2+}$ overload in HT22 cells after sevoflurane exposure.

There were some limitations in this study. Firstly, it was only in vitro. However, this study is just the first step to testify our hypothesis. On the basis of this study, we will perform the further experiments to testify the signaling pathway in vivo. Secondly, WNK463 is a specific compound. It is still far from being used as a protective agent in vivo. However, the results of this study will help us to understand the possible mechanism of this pathway in sevoflurane neurotoxicity. So as to provide new ideas for us to find more practical and transformable protective agents on the WNK1/NKCC1/GABA ${ }_{A} R$ pathway.

\section{Conclusion}

The results of this study indicate that sevoflurane can inhibit the proliferation of HT22 cells and induce cell apoptosis, while WNK1 kinase antagonist can reverses these changes and produces protective effects. The WNK1/NKCC1 signalling pathway plays an important role. Reducing intracellular calcium influx may be a change in the downstream of this signalling pathway.

\section{List Of Abbreviations}


$\mathrm{GABA}_{\mathrm{A}} \quad \mathrm{Y}$-aminobutyric acid type A receptor

NMDA N-methyl-D-aspartate

WNKs with-no-lysine

\begin{tabular}{ll} 
NKCC1 & sodium-potassium-chloride cotransporter-1 \\
\hline KCC2 & potassium-chloride cotransporter-2 \\
\hline DMEM & Dulbecco's Modified Eagle's Medium Gibco
\end{tabular}

\begin{tabular}{ll}
\hline SDS & Sodium dodecyl sulfate \\
\hline Tris & Trishydroxymethylaminomethane \\
\hline PAGE & Polyacrylamine electrophoresis gel \\
\hline PVDF & polyvinylidene fluoride \\
\hline BCA & Bicinchoninic acid \\
\hline PBS & Phosphate buffered saline
\end{tabular}

\section{Declarations}

\section{Ethics approval and consent to participate}

Not applicable.

\section{Consent for publication}

Not applicable.

\section{Availability of data and materials}

Not applicable.

\section{Competing interests}

The authors declare that they have no competing interests.

\section{Funding}


This work was supported by the Research Foundation of Beijing Friendship Hospital, Capital Medical University [grant numbers yyqdkt2018-23].

\section{Authors' contributions}

All authors contributed to the study conception and design. ALX was responsible for quality control of the design, project implementation and paper revision. Material preparation, data collection and analysis were performed by BYF, LWJ. ZL was responsible for western-blot experiment. LJF directed the completion of the experiment. All authors read and approved the final manuscript.

\section{Acknowledgements}

I would like to thank the department of Neurobiology and Beijing Institute for Brain Disorders of the Capital Medical University for its strong support and the hard work of all the experimenters.

\section{References}

1. Rappaport BA, Suresh S, Hertz S, et al. Anesthetic neurotoxicity-clinical implications of animal models. N Engl J Med. 2015;372:976-7.

2. Ikonomidou C, Bosch F, Miksa M, et al. Blockade of nmda receptors and apoptotic neurodegeneration in the developing brain. Science.1999; 283:70-4.

3. Zheng SQ, An LX, Chen X, Wang Y. Sevoflurane Causes Neuronal Apoptosis and Adaptability Changes of Neonatal Rats. Acta Anaesth Scand. 2013; 57:1167-74.

4. Han XD, Li M, Zhang XG, et al. Single sevoflurane exposure increases methyl-CpG island binding protein2 phosphorylation in the hippocampus of developingmice. Mol Med Rep. 2015; 11:226-30.

5. Briner A, De Roo M, Dayer A, et a1. Volatile anesthetics rapidly increase dendritic spine density in the rat medial prefrontal cortex during synaptogenesis. Anesthesiology. 2010; 112:546-56.

6. Andropoulos DB, Greene MF. Anesthesia and Developing Brains - Implications of the FDA Warning. N Engl J Med. 2017; 376:905-7.

7. Xia Y, Xu H, Jia C, et al. Tanshinone IIA Attenuates Sevoflurane Neurotoxicity in Neonatal Mice. Anesth Analg. 2017; 124:1244-52.

8. Ju X, Jang Y, Heo JY, et al. Anesthesia affects excitatory/inhibitory synapses during the critical synaptogenic period in the hippocampus of young mice: Importance of sex as a biological variable. Neurotoxicology. 2019; 70:146-53.

9. McCann ME, de Graaff JC, et al. Neurodevelopmental outcome at 5 years of age after general anaesthesia or awake-regional anaesthesia in infancy (GAS): an international, multicentre, randomised, controlled equivalence trial. Lancet. 2019;393:664-77.

10. Davidson AJ, Disma N, de Graaff JC, et al. Neurodevelopmental outcome at 2 years of age after general anaesthesia and awake-regional anaesthesia in infancy (GAS): an international multicentre, randomised controlled trial. Lancet. 2016; 387:293-50. 
11. Sun LS, Li G, Miller TL, et al. Association between a single general anesthesia exposure before age 36 moths and neurocognitive outcomes in later childhood. JAMA. 2016; 315:2312-20.

12. Gleich SJ, Flick R, Hu D, Zaccariello MJ. Neurodevelopment of children exposed to anesthesia: design of the Mayo Anesthesia Safety in Kids (MASK) study. Contemp Clin Trials. 2015; 41:45-54.

13. Pontes $A$, Zhang $Y$, Hu W. Novel functions of GABA signaling in adult neurogenesis. Front Biol(Beijing). 2013;8:496-507.

14. Kotani N, Akaike N. The effects of volatile anesthetics on synaptic and extrasynaptic GABA-induced neurotransmission. Brain Res Bull. 2013; 93:69-79.

15. Ben-Ari Y, Khalilov I, Kahle KT, Cherubini E. The GABA excitatory/inhibitory shift in brain maturation and neurological disorders. Neuroscientist. 2012; 18:467-86.

16. Rivera $\mathrm{C}$, Voipio J, Payne JA, et al. The $\mathrm{K}^{+} / \mathrm{Cl}^{-}$co-transporter $\mathrm{KCC} 2$ renders GABA hyperpolarizing during neuronal maturation. Nature. 1999; 397:251-255.

17. Friedel P, Kahle KT, Zhang J, et al. WNK1regulated inhibitory phosphorylation ofthe KCC2 cotransporter maintains the depolarizing action of GABA in immature neurons. Sci Signal. 2015; 30:ra65.

18. Xie SN, Ye Hong, Li JF, An LX. Sevoflurane Neurotoxicity in Neonatal Rats is Related to an Increase in the GABAAR a1/ GABAAR a2 Ratio. J Neuro Res. 2017; 95:2367-75.

19. Stevens RA, Butler BD, Kokane SS, et al. Neonatal inhibition of $\mathrm{Na}^{+}-\mathrm{K}^{+}-2 \mathrm{Cl}^{-}$cotransporter prevents ketamine induced spatial learning and memory impairments. Neurotoxicol Teratol. 2017; 60:82-6.

20. Li Y, Xia H, Chen L, Zhang X. Sevoflurane induces endoplasmic reticulum stress mediated apoptosis in mouse hippocampal neuronal HT22 cells via modulating miR-15b-5p/Rab1A signaling pathway. Int J Clin Exp Pathol. 2017;10:8270-80.

21. Zhang L, Zhang J, Yang L, Dong Y. Isoflurane and sevoflurane increase interleukin-6 levels through the nuclear factor-kappa B pathway in neuroglioma cells. Br J Anaesth. 2013;110:82-91.

22. Liu Q, Wang G, Chen Y, et al. A miR-590/ACVR2a/Rad51B axis regulates DNA damage repair during mES cell proliferation. Stem Cell Reports. 2014;3:1103-17.

23. Bai X, Yan Y, Canfield S, et al. Ketamine enhances human neural stem cell proliferation and induces neuronal apoptosis via reactive oxygen species-mediated mitochondrial pathway. Anesth Analg. 2013;116:869-80.

24. Cui Y, Ling-Shan G, Yi L, et al. Repeated administration of propofol upregulated the expression of cFos and cleaved-caspase-3 proteins in the developing mouse brain. Indian J Pharmacol. 2011;43:648-51.

25. Clayton G, Owens G, Wolff J, Smith R. Ontogeny of cation $\mathrm{Cl}^{-}$cotransporter expression in rat neocortex. Dev Brain Res. 1998; 109:281-92.

26. Chamma I, Chevy Q, Poncer JC, et al. Role of the neuronal K-Cl cotransporter KCC2 in inhibitory and excitatory neurotransmission. Front Cell Neurosci. 2012;6:5. 
27. Gagnon KB, England R, Delpire E. Volume sensitivity of cation- $\mathrm{Cl}^{-}$cotransporters is modulated by the interaction of two kinases: Ste20-related proline- alanine-rich kinase and WNK4. Am J Physiol Cell Physio. 2006;290:c134-42.

28. Friedel P, Kahle KT, Zhang J, et al. WNK1-regulated inhibitory phosphorylation of the KCC2 cotransporter maintains the depolarizing action of GABA in immature neurons. Sci Signal. 2015; 30:ra65.

29. Heubl M, Zhang J, Pressey JC, et al.

GABAA receptor dependent synaptic inhibition rapidly tunes KCC2 activity via the $\mathrm{Cl}^{-}$ sensitive WNK1 kinase. Nat Commun. 2017;8:1776-92.

30. Yang M, Wei H. Anesthetic neurotoxicity: Apoptosis and autophagic cell death mediated by calcium dysregulation. Neurotoxicol Teratol. 2017;60:59-62.

\section{Figures}

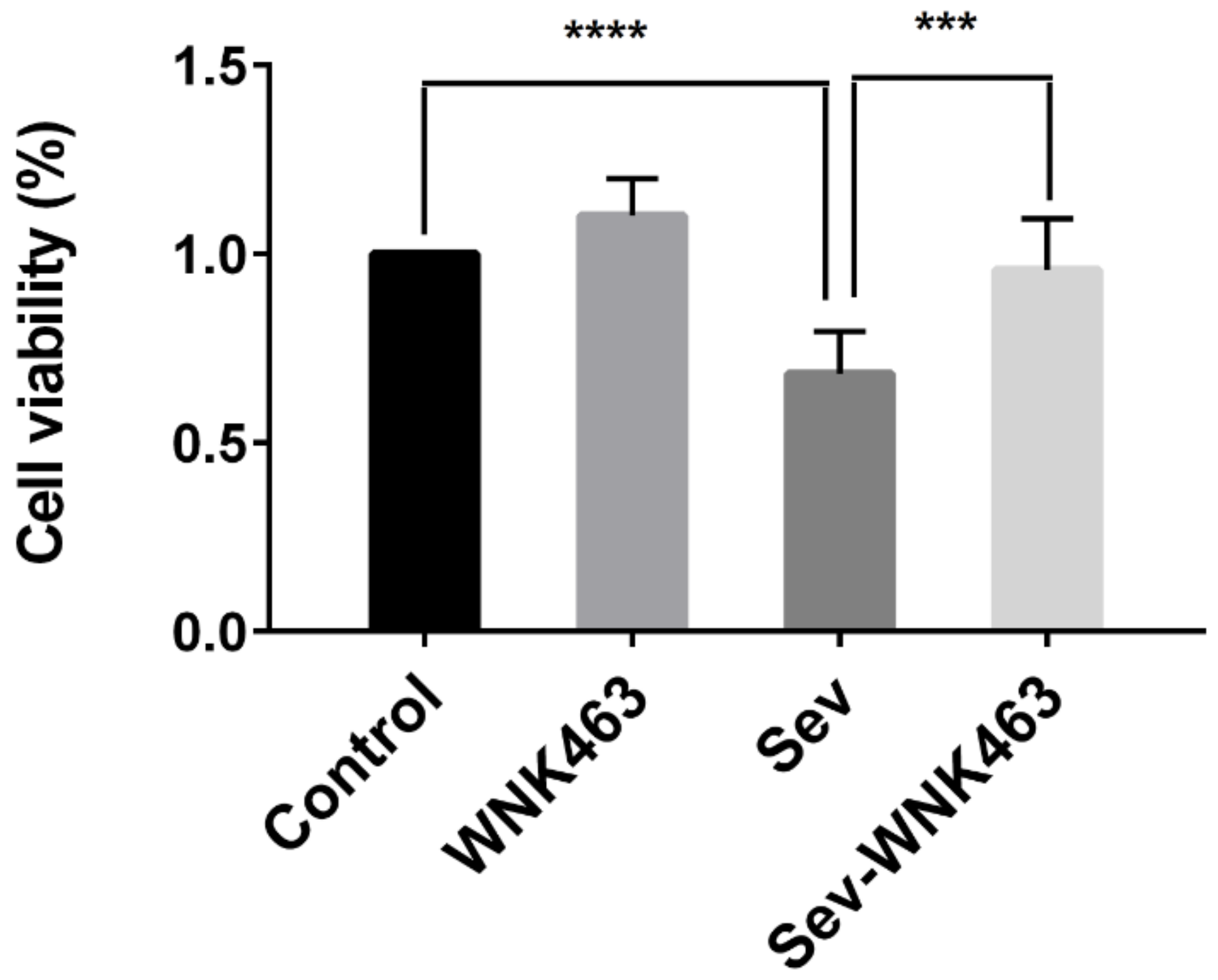




\section{Figure 1}

WNK-463 alleviated sevoflurane-induced cell activity decreasing Figure 1. The MTS was used to measure cell activity following treatment with $0 \%$ or $4.1 \%$ Sev for $6 \mathrm{~h}$ or added to $1 \mu \mathrm{mol}$ WNK-463 (The data represent the mean $\pm S D, n=6, * \star * P<0.001)$.
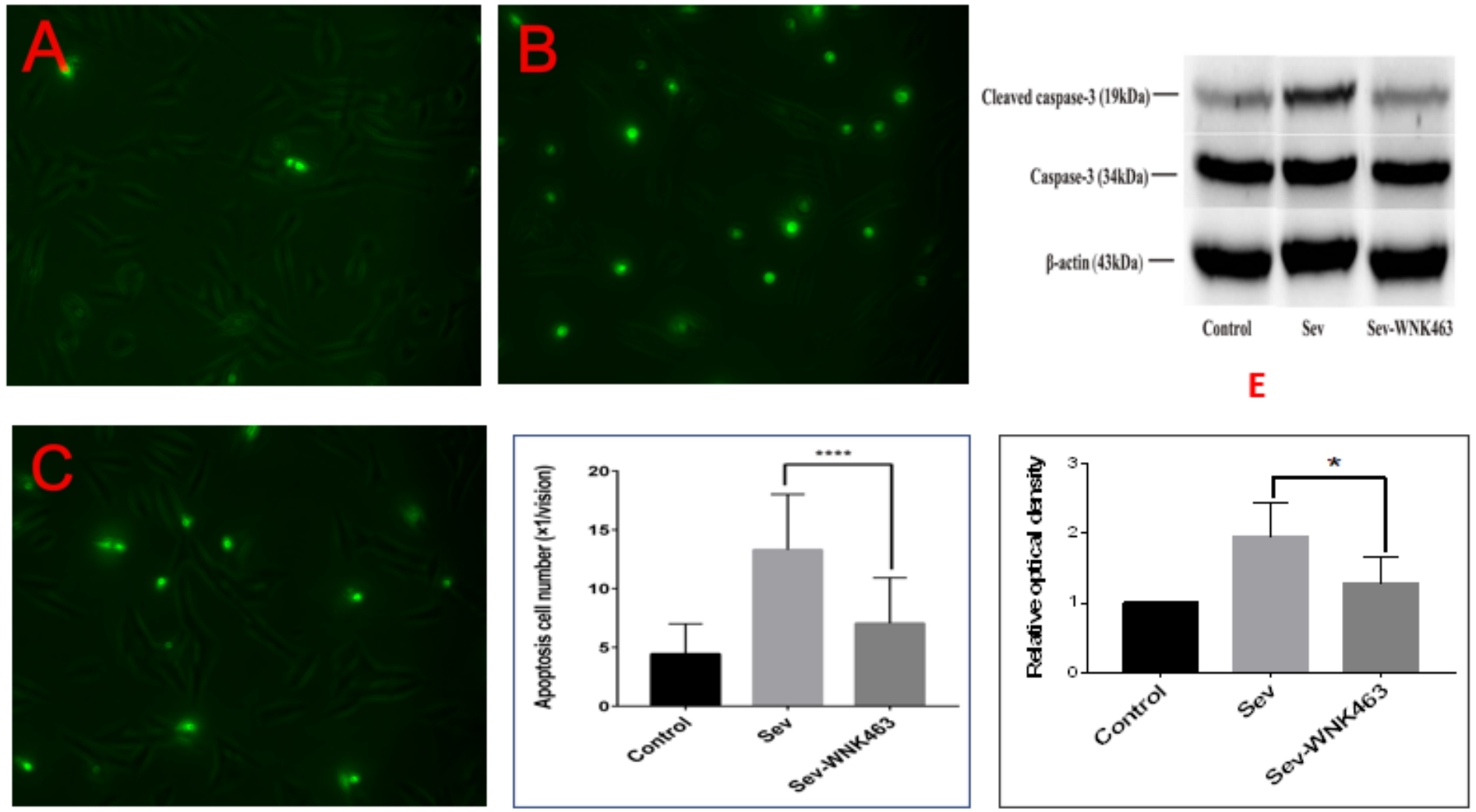

D

$\mathbf{F}$

\section{Figure 2}

WNK-463 decreased HT22 cell apoptosis caused by sevoflurane Figure 2. (A) TUNEL staining was performed on the Control HT22 cells; (B) TUNEL staining was performed on the HT22 cells exposed to $4.1 \%$ sevoflurane for 6 h; (C) Given WNK-463, TUNEL staining on the HT22 cells exposed to sevoflurane for 6 h; (D) Compared the apoptosis cell numbers among control, sevoflurane treatment and WNK-463+ sevoflurane treatment HT22 cells. Compared with the control group, sevoflurane increased the number of apoptosis cells. And WNK-463 decreased the apoptosis cells number compared with sevoflurane group $(\star \star \star \star P<0.0001)$. (E) After exposure to sevoflurane, with or without WNK-463, the cleaved-caspase-3 and caspase-3 protein levels were measured using Western blot analysis. $\beta$-actin was used as an internal control; (F) Quantification of cleaved caspase-3 and caspase-3 expression at three replications. (The data represent the mean $\pm S D, n=6, * P<0.05)$. 

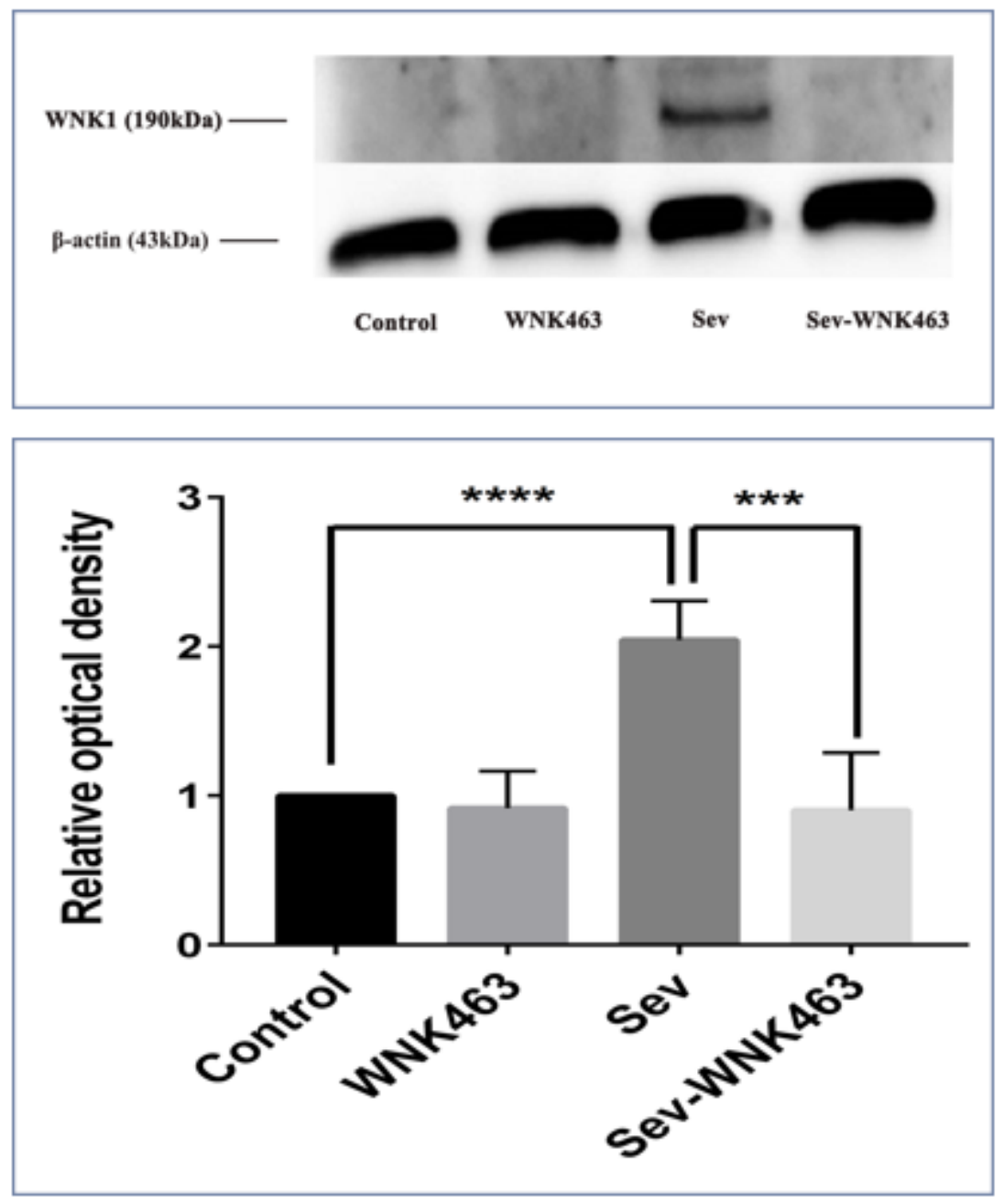

\section{Figure 3}

WNK-463 inhibited WNK1 kinase concentration increasing caused by sevoflurane Figure 3. (A) The WNK1 kinase protein levels measured using wester western blot analysis. $\beta$-actin was used as an internal control. (B) Quantification of WNK1 kinase expression at four groups. The data represent the mean \pm SD based on the gray value of the control group, standardized analysis was performed. $(\star \star \star P<0.001)$. 

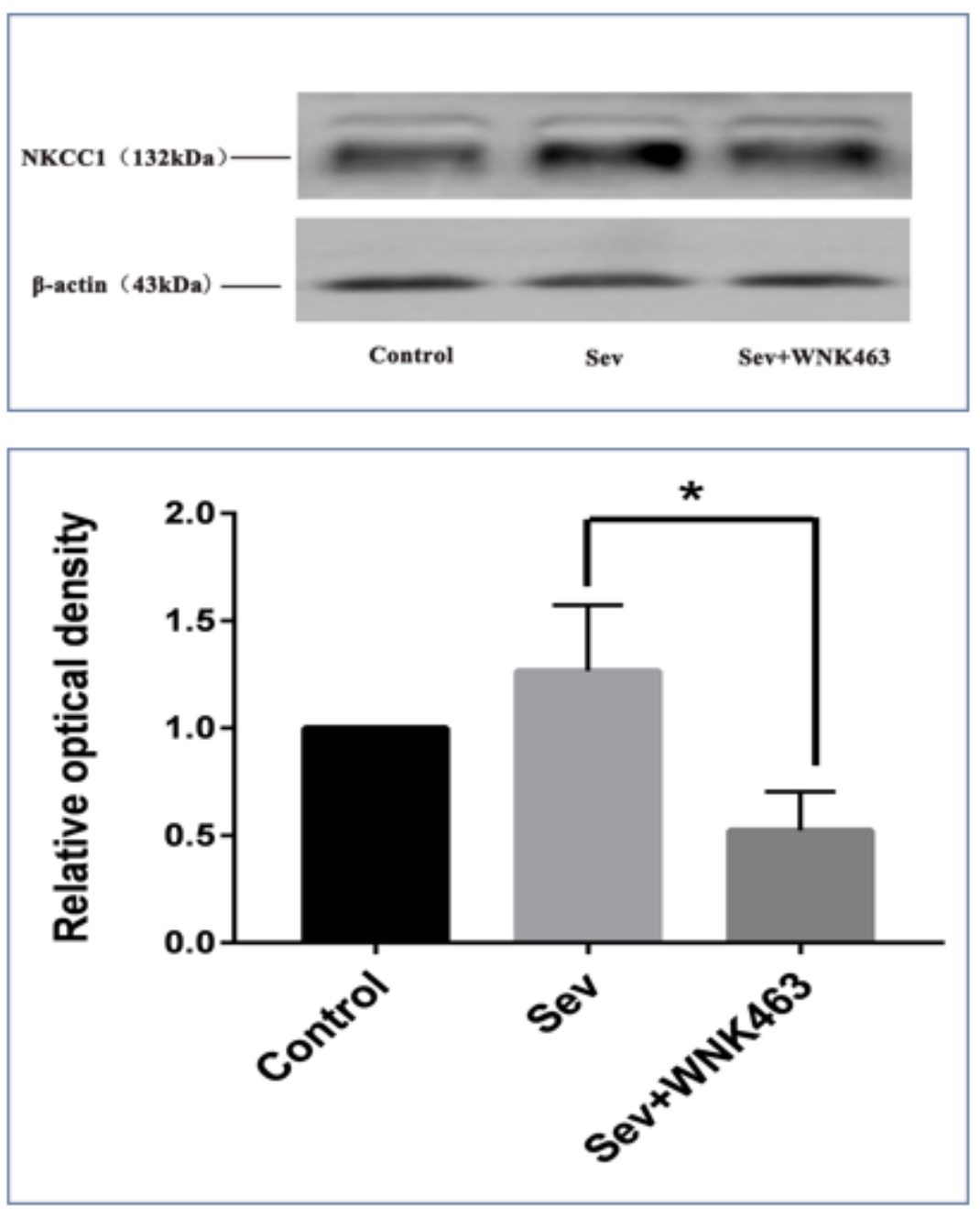

\section{Figure 4}

WNK-463 inhibited NKCC1 increasing caused by sevoflurane Figure 4. (A) The NKCC1 protein levels measured using wester western blot analysis. $\beta$-actin was used as an internal control. (B) Quantification of NKCC1 expression at four groups. The data represent the mean \pm SD based on the gray value of the control group, standardized analysis was performed. ( $\left.{ }^{\star} \mathrm{P}<0.05\right)$. 

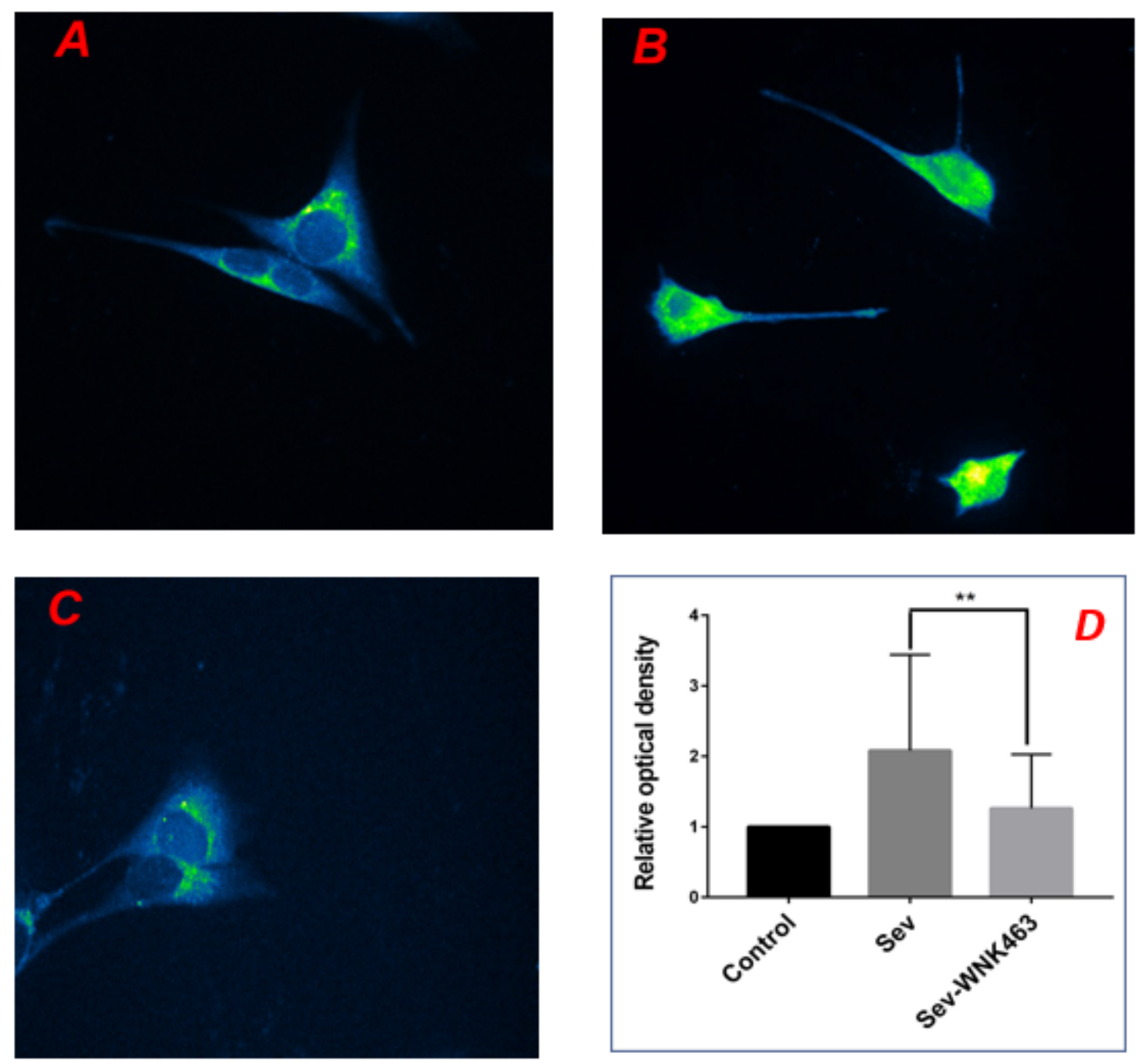

\section{Figure 5}

WNK-463 reduced the calcium overload in HT22 cells after exposure to sevoflurane Figure5. (A) The fluo4 Calcium ion imaging technology was performed on the Control HT22 cells; (B) Exposed to 4.1\% sevoflurane for 6 h; (C) Given WNK-463, on the HT22 cells exposed to sevoflurane for 6 h; (D) Quantification of calcium ion imaging at three groups. The data represent the mean \pm SD based on the gray value of the control group, standardized analysis was performed. ( $* \star P<0.01)$

\section{Supplementary Files}

This is a list of supplementary files associated with this preprint. Click to download.

- SupplementaryFiles.docx 